Pacific Journal of Mathematics

DUALITY IN GENERAL ERGODIC THEORY 


\title{
DUALITY IN GENERAL ERGODIC THEORY
}

\author{
ROBERT G. HEYNEMAN
}

Introduction. Let $G$ be a semi-group of operators acting on a Banach space $E$. Alaoglu-Birkhoff [1], Eberlein [8], Jacobs [9], deLeeuw and Glicksberg [12], and others have given conditions under which certain orbits (see $\S 1$ ) in $E$ will contain a single fixed vector under the action of $G$. In general of course, a given orbit may contain many fixed points or none at all; moreover it need not be the case that the 'ergodic' vectors (those whose orbits contain a single fixed point) form a linear subspace as one would wish.

The object of this paper is to show how the introduction of considerations involving the conjugate space $E^{*}$ under the action of the adjoint semi-group $G^{*}$ illuminate these matters. We shall see that there is an intimate connection between the existence of fixed points in the orbits of one space and the uniqueness of fixed points in the orbits of the associated space. Our first result in this direction, Theorem 1.3, asserts that if every orbit in one space contains at least one fixed point, then every orbit in the other contains at most one fixed point.

In $\S 2$ we define what we mean by saying that the semigroup $G$ acts ergodically on the space $E$. When this is the case the pathology that arises from the existence of more than one fixed point in a given orbit of $E$ cannot occur. Thus the ergodicity of $G$ on $E$ may be considered as a strong uniqueness requirement on the fixed points of orbits of $E$. When $E$ is reflexive we can then show that this requirement (that $(G, E$ ) is ergodic) may be characterized by the fact that every orbit of the conjugate space $E^{*}$ contains at least one fixed point under the adjoint semigroup $G^{*}$. Indeed whether $E$ is reflexive or not, Theorem 3.1 asserts that the 'ergodic behaviour' of the orbits of one space insures the existence of (at least) one fixed point in any weakly compact orbit of the other.

These results 'explain' and unify many earlier results which were obtained using different specialized techniques. The following two examples are instructive:

(a) $G$ is abelian. As it is quite trivial to verify that abelian semigroups act ergodically, both $(G, E)$ and $\left(G^{*}, E^{*}\right)$ are ergodic. Then since $(G, E)$ is ergodic (respectively $\left(G^{*}, E^{*}\right)$ is ergodic), we see that every weakly compact orbit of $E$ contains at most (respectively at least) one fixed point. Thus weakly compact orbits contain precisely one fixed point (cf. for example [8]).

(b) $G$ is a group acting on a Hilbert space $E$. Here one can show

Received June 2, 1962. This work was supported by the Office of Ordnance Research (Contract DA-04-200-ORD-171). 
(Jacobs [9]) that whenever a (bounded) group acts on a Hilbert space, any orbit contains at least one fixed point. Since this applies to $\left(G^{*}, E^{*}\right)$ as well as to $(G, E)$, we see from the previous discussion that every orbit of $E$ contains exactly one fixed point. Jacobs makes use of the special nature of the hypotheses on $G$ and $E$ in deriving the uniqueness of fixed points in orbits in case (b). Nonetheless the spirit of his argument is akin to ours and suggested the interest of such an investigation as the present one.

In the last section of the paper we describe the relationship between ergodicity and invariant means. We show in particular that if $(G, E)$ is ergodic where $G$ is a semi-group of 'transition operators' on an appropriate space $E$, then $E$ admits a mean which is invariant under $G$.

This paper represents part of the authors doctoral dissertation presented in 1957 at the University of California at Berkeley. In the authors thesis ergodicity was also characterized generally in terms of the notion of convergence due to Birkhoff and Alaoglu [1]. Here we have preferred to proceed independently of all convergence considerations in an entirely self-contained way. The author would like to thank Professor F. Wolf, under whose direction the thesis was written for his generous help and advice.

1. Fixed points in orbits. Throughout this paper $G$ will denote a bounded semi-group of linear transformations acting on a Banach space $E$. This means simply that $G$ is closed under multiplication and that there is a positive number $M$ such that $\|g x\|<M\|x\|$ for all $x \in E$ and $g \in G$. We will assume that $G$ contains the identity transformation. If $x \in E$, the closed convex hull of the set $\{g x ; g \in G\}$ will be referred to as the orbit of $x$ and denoted $K(x)$; subsets of this type will frequently be called orbits without specific reference to the generating vector. $\boldsymbol{G}$ will denote the collection of operators on $E$ which are convex combinations of elements of $G$. Then $\boldsymbol{G}$ is a bounded semi-group in its own right with the same bound $M$, the same orbits, and the same fixed points as $G$. Clearly $K(x)=$ closure $\{\boldsymbol{g} x ; \boldsymbol{g} \in \boldsymbol{G}\}$. Finally we define $N=\{x \in E ; 0 \in K(x)\}$, $F=\{x \in E ; g x=x$ for all $g \in G\}, D=\{x-g x ; x \in E$ and $g \in G\}$, and $[D]=$ the closed subspace spanned by $D$.

In passing to the action of the adjoint semi-group $G^{*}$ on the conjugate space $E^{*}$, the corresponding dual objects are naturally defined. Thus if $\xi \in E^{*}$, the orbit of $\xi$ will mean the closed convex hull of $\left\{g^{*} \xi ; g^{*} \in G^{*}\right\}$ and will be denoted again $K(\xi)$. In the same spirit we define $G^{*}, N_{*}, D_{*}$ and $F_{*}$.

We use the notation $(x, \xi)$ to express the linkage between a vector $x \in E$ and a vector $\xi \in E^{*}$. If $S$ is a subset of $E$ and $T$ is a subset of $E^{*}$, we set $S^{\perp}=\left\{\xi \in E^{*} ;(x, \xi)=0\right.$ for all $\left.x \in S\right\}$ and $T^{\perp}=\{x \in E ;(x, \xi)=0$ for all $\xi \in T\}$. Recall that $S^{\perp \perp}=[S]$, the closed subspace spanned by $S$. 
The following technical proposition expressing the relationships between the sets we have defined will be used repeatedly throughout this paper.

\subsection{Proposition.}

1.1.1. $N \cap F=0$

1.1.2. $D^{\perp}=F_{*}$ and $D_{*}^{\perp}=F$

1.1.3. $N$ is closed in $E$

1.1.4. $D \subset N \subset[D]$

1.1.5. If $x_{0} \in F$, then $x_{0} \in K(x)$ if and only if $x-x_{0} \in N$

1.1.6. If $[D] \cap F=0$, then any orbit of $E$ can contain at most one fixed point.

Proof. If $x \in N \cap F$, then $K(x)=\{x\}$ and also $0 \in K(x)$ so that (1) follows. (2) is immediate by virtue of the identity $(x-g x, \xi)=\left(x, \xi-g^{*} \xi\right)$. To prove (3), let $x \in \bar{N}$ and choose $n \in N$ with $\|x-n\|<\varepsilon$. We can then find $\boldsymbol{g} \in \boldsymbol{G}$ with $\|\boldsymbol{g} n\|<\varepsilon$. We now have $\|\boldsymbol{g} \boldsymbol{x}\| \leqq\left\|\boldsymbol{g}(x-n)^{\prime}\right\|+$ $\|\boldsymbol{g} n\|<(M+1) \varepsilon$ so that $x \in N$.

To prove (4), we define $g_{n}=1 / n\left(1+g+g^{2}+\cdots+g^{n-1}\right)$ where $g \in G$. Then $\boldsymbol{g}_{n} \in \boldsymbol{G}$. If now $x-g x \in D$, we have $\boldsymbol{g}_{n}(x-g x)=1 / n\left(x-g^{n} x\right) \rightarrow 0$ so that $0 \in K(x-g x)$. Thus $D \subset N$. To show that $N \subset[D]$, it will suffice to prove that $\left(N, F_{*}\right)=0$ for then $N \subset F_{*}^{\perp}=D^{\perp \perp}=[D]$. But if $n \in N$ and $\xi \in F_{*}$ we may choose $\boldsymbol{g} \in \boldsymbol{G}$ with $\|\boldsymbol{g} n\|<\varepsilon /\|\xi\|$. We have:

$$
|(n, \xi)|=\left|\left(n, g^{*} \xi\right)\right|=|(g n, \xi)| \leqq\|\boldsymbol{g} n\|\|\xi\|<\varepsilon
$$

so that

$$
(n, \xi)=0 .
$$

If $x_{0} \in F$, then $g x-x_{0}=g\left(x-x_{0}\right)$ so that $\left\|g x-x_{0}\right\|<\varepsilon$ if and only if $\left\|\boldsymbol{g}\left(x-x_{0}\right)\right\|<\varepsilon$. This proves (5).

To prove (6), let $x_{1}, x_{2}$ be fixed points in the orbit $K(x)$. Then by (5) $n_{1}=x-x_{1}$ and $n_{2}=x-x_{2}$ are both in $N$. Since $N \subset[D]$, this means that $n_{1}$ and $n_{2}$ are in $[D]$ so that $n_{1}-n_{2}=x_{2}-x_{1} \in[D] \cap F$. In particular if $[D] \cap F=0$, then $x_{1}=x_{2}$.

1.2 ExAmple. In the classical context where $G$ is the bounded semigroup consisting of the powers of a single operator $T$ (where $\left\|T^{k}\right\| \leqq M$, $k=1,2, \cdots)$, we may identify $N$ with the closed subspace: $\eta=\{x \in E$; $\left.T_{n} x=1 / n\left(x+T x+\cdots+T^{n-1} x\right) \rightarrow 0\right\}$. For, take $x-T^{k} x \in D$. Then $T_{n}\left(x-T^{k} x\right)=k / n T_{k}\left(1-T^{n}\right) x \rightarrow 0$ so that $D \subset \eta$. Since $\eta$ is closed, this means that $[D] \subset \eta$ and so $N \subset \eta$. But also if $T_{n} x \rightarrow 0$, then $0 \in K(x)$ so that $\eta \subset N$. Thus $N=\eta$.

If now $x \in E$ and $x_{0}$ is a fixed point in $K(x)$ then by (1.5) $x-x_{0} \in N$ and so $T_{n} x-x_{0}=T_{n}\left(x-x_{0}\right) \rightarrow 0$. Consequently $T_{n} x \rightarrow x_{0}$. We have 
thus shown: if the orbit of a vector $x \in E$ contains a fixed point $x_{0}$, then $T_{n} x$ converges to $x_{0}$. Conversely the identity $(1-T) T_{n} x=1 / n\left(1-T^{n}\right) x$ shows that if $T_{n} x$ converges to $x_{0}$, then $x_{0}$ is a fixed point. (cf. Eberlein [8]).

1.3 THEOREM. If every orbit of $E$ (respectively $E^{*}$ ) contains at least one fixed point, then any orbit of $E^{*}$ (respectively $E$ ) contains at most one fixed point.

Proof. If the orbit of every vector $x \in E$ contains a fixed point $x_{0}$, then $x-x_{0} \in N$, so that every vector $x$ in $E$ can be expressed as the sum of a vector $x_{0}$ in $F$ and a vector $x-x_{0}$ in $N$. Thus $F^{\perp} \cap N^{\perp}=0$. Now since $D \subset N \subset[D]$ one has $N^{\perp}=D^{\perp}=F_{*}$. Also $\left(F, D_{*}\right)=0$ so that $F^{\perp}$ contains $\left[D_{*}\right]$. Consequently $F^{\perp} \cap N^{\perp}$ contains $\left[D_{*}\right] \cap F_{*}$ and so $\left[D_{*}\right] \cap F_{*}=0$. Applying Proposition 1.1.6 to the adjoint space, the conclusion then follows.

If every orbit of $E^{*}$ contains at least one fixed point, then by what we have just shown, any orbit of $E^{* *}$ contains at most one fixed point. But because of the isometric imbedding of $E$ in $E^{* *}$, the orbit of a vector $x \in E$ is the same whether $x$ is considered to lie in $E$ or $E^{* *}$. Thus orbits of $E$ contain at most one fixed point.

1.3.1 CoROllary. If every orbit in $E$ and in $E^{*}$ contains at least one fixed point, then any orbit in $E$ or in $E^{*}$ contains precisely one fixed point.

\subsection{EXAMPLeS}

1.4.1. If $G$ consists of contractions ${ }^{1}$ on Hilbert space then any orbit $K(x)$ certainly contains at least one fixed point. For if $x_{0}$ is the (unique) element of $K(x)$ having smallest norm, then since $\left\|g x_{0}\right\| \leqq\left\|x_{0}\right\|$ and $g x_{0} \in K(x)$, it follows from the defining property of $x_{0}$ that $g x_{0}=x_{0}$; that is, $x_{0}$ is a fixed point.

As the same argument applies to the adjoint semi-group $G^{*}$ (which also consists of contractions on Hilbert space) we conclude by Corollary 1.3.1 that every orbit contains precisely one fixed point (Alaoglu-Birkhoff [1]).

1.4.2. More generally Day [5], pointed out that whenever $G$ consists of contractions on a strictly-convex ${ }^{2}$ reflexive space, the above argument is still effective and shows that every orbit contains at least one fixed

1 An operator $T$ is called a contraction if $\|T\| \leqq 1$. It is called an isometry if $\|T x\|=$ $\|x\|$ for all $x \in E$.

2 A Banach space is strictly convex is the unit sphere (vectors of norm 1) contains no line segment. 
point. Thus if we assume that both $E$ and $E^{*}$ are strictly convex, Corollary 1.3.1 again allows us to conclude that every orbit contains precisely one fixed point. Such is the case, for example, if $E$ is an $L_{p}$ space for $p>1$.

1.4.3. If $G$ consists of a (bounded) group acting on a Hilbert space $E_{1}$ then we may define a new norm on $E$ in the following way: $|x|^{2} \equiv$ $\sup _{g \in G}\langle g x, g x\rangle$. This clearly defines an equivalent norm relative to which $G$ acts isometrically. Moreover Jacobs [9] has shown that this new norm is strictly convex. Thus by Day's result, every orbit of $E$ contains at least one fixed point. Since $G^{*}$ is also a group, the same conclusion is valid for orbits of $E^{*}$; by Corollary 1.3.1 it then follows that every orbit of $E$ contains precisely one fixed point (Jacobs [9]).

1.5. REMARK. We are indebted to the referee for informing us of some unpublished results of C. Ryll-Nardzewski [14]. His results imply the following: if $G$ is a semi-group of isometries ${ }^{1}$ on a Banach space $E$ then any weakly-compact orbit of $E$ contains fixed points. In particular if $E$ is reflexive (and $G$ consists of isometries) then every orbit of $E$ contains at least one fixed point. If in addition $G^{*}$ also acts as isometries on the (reflexive) space $E^{*}$, we conclude by Corollary 1.3.1 that every orbit of $E$ contains precisely one fixed point.

In the same way if $G$ is any (bounded) group acting on a reflexive Banach space we may renorm the space as in Example 3 above so that $G$ consists of isometries. The result of Ryll-Nardzewski thus again applies to show that in this case too every orbit contains precisely one fixed point

2. Ergodicity and duality. We now proceed to an examination of the 'good' case where $G$ acts 'ergodically' on $E$.

2.1 Proposition. The following conditions are equivalent:

1. $\boldsymbol{g} N \subset N$ for any $\boldsymbol{g} \in \boldsymbol{G}$ (if $0 \in K(n)$, then $0 \in K(\boldsymbol{g} n)$ ).

2. If $n \in N$ then $K(n) \subset N$.

3. $N$ is a linear subspace of $E$ (i.e. if $0 \in K(x)$ and $0 \in K(y)$, then $0 \in K(x+y))$.

4. $N=[D]$.

Proof. (1) $\Longrightarrow(2)$ since $N$ is closed.

(2) $\Longrightarrow$ (3). Let $x \in N$ and $y \in N$. Choose $g_{1} \in \boldsymbol{G}$ such that $\left\|\boldsymbol{g}_{1} x\right\|<\varepsilon$. Then as $\boldsymbol{g}_{1} y \in N$ by (2), we can choose $\boldsymbol{g}_{2} \in \boldsymbol{G}$ such that $\left\|\boldsymbol{g}_{2} \boldsymbol{g}_{1} y\right\|<\varepsilon$. We now have: $\left\|\boldsymbol{g}_{2} \boldsymbol{g}_{1}(x+y)\right\| \leqq M\left\|\boldsymbol{g}_{1} x\right\|+\left\|\boldsymbol{g}_{2} \boldsymbol{g}_{1} y\right\|<(M+1) \varepsilon$. Thus $x+y \in N$.

(3) $\Longrightarrow$ (4) for $N$ is closed and $D \subset N \subset[D]$. 
(4) $\Longrightarrow(1)$. Since $F_{*}$ is invariant under the action of $G^{*}$, it follows that $[D]=F_{*}^{+}$is invariant under the action of $G$. In particular, if $[D]=N$, then condition (1) is satisfied.

The pair $(G, E)$ will be called ergodic if any of the above conditions is satisfied. We will then call a vector of $E$ ergodic if its orbit contains a fixed point. By proposition 1.1.5 and proposition 2.1 a vector $x \in E$ is ergodic if and only if it belongs to the subspace $R=N \oplus F=[D] \oplus F$. $R$ will be referred to as the ergodic subspace. (This nomenclature is in accord with a somewhat unfortunate tradition.)

2.2 ExAmples 2.2.1. If $G$ is abelian, then $(G, E)$ is ergodic. For if $n \in N$ and $\boldsymbol{g} \in \boldsymbol{G}$ we may choose $\boldsymbol{g}_{1} \in \boldsymbol{G}$ with $\left\|\boldsymbol{g}_{1} n\right\|<\varepsilon$. We then have $\left\|\boldsymbol{g}_{1} \boldsymbol{g} n\right\|=\left\|\boldsymbol{g} \boldsymbol{g}_{1} n\right\|<M \varepsilon$ so that $\boldsymbol{g} n \in N$. Thus $(G, E)$ is ergodic by proposition 2.1.1.

2.2.2. If every orbit of $E$ contains precisely one fixed point, then $(G, E)$ is ergodic. For let $n \in N$ and $\boldsymbol{g} \in \boldsymbol{G}$. Since $K(\boldsymbol{g} n) \subset K(n)$, the fixed point of $K(\boldsymbol{g} n)$ must coincide with that of $K(n)$; that is, $0 \in K(\boldsymbol{g} n)$. Thus $g n \in N$ and so again by proposition 2.1.1 $(G, E)$ is ergodic.

2.2.3. If $G$ admits a right invariant mean, then $(G, E)$ is ergodic (Theorem 4.2).

2.2.4. If every orbit of $E^{*}$ contains at least one fixed point, then $(G, E)$ is ergodic. (We will see later-cf. Corollary 3.1.1-that when $E$ is reflexive, $(G, E)$ is ergodic if and only if every orbit of $E^{*}$ contains at least one fixed point.)

Proof. If $(G, E)$ is not ergodic, by proposition 2.1.1 there is an $n \in N$ and a $\boldsymbol{g} \in \boldsymbol{G}$ with $\boldsymbol{g} n \notin N$, so that $0 \in K(n)$ but $0 \notin K(\boldsymbol{g} n)$. The Hahn-Banach Theorem then asserts the existence of a functional $\xi \in E^{*}$ which separates 0 from the closed convex set $K(\boldsymbol{g} n)$; that is, $0<\alpha \leqq$ $(K(\boldsymbol{g} n), \xi)$ where $\alpha$ is a real number. In particular, $0<\alpha \leqq(\boldsymbol{G g} n, \xi)=$ $\left(\boldsymbol{g} n, \boldsymbol{G}^{*} \xi\right)$ and so $0<\alpha \leqq\left(\overline{\left.g n, \boldsymbol{G}^{*} \xi\right)}\right.$. But as $\boldsymbol{g} n$ induces a continuous functional on $E^{*}$ (in the norm topology on $E^{*}$ ) we have:

$$
\left(\overline{\boldsymbol{g} n, \boldsymbol{G}^{*} \xi}\right) \supset\left(\boldsymbol{g} n,\left\{\overline{\boldsymbol{G}^{*} \xi}\right\}\right)=(\boldsymbol{g} n, K(\xi))
$$

so that $0<\alpha \leqq(g n, K(\xi))$. If then $K(\xi)$ were to contain a fixed point $\xi_{0}$, we would have $0<\alpha \leqq\left(g n, \xi_{0}\right)=\left(n, \xi_{0}\right)$ which would contradict the fact that $N$ is perpendicular to $F_{*}$.

2.3 Theorem. If $(G, E)$ is ergodic, then:

2.3.1. the ergodic subspace $R$ is closed and strongly invariant (in 
the sense that $g x \in R$ if and only if $x \in R$ ),

2.3.2. the orbit of a vector $x \in E$ contains precisely one fixed point if $x \in R$ and contains none if $x \notin R$,

2.3.3. if $x \in R$ and $p(x)$ is the associated fixed point of $K(x)$ then $x \rightarrow p(x)$ defines a (bounded) linear operator $p$ on $R$ such that $p g=$ $g p=p^{2}=p .^{3}$

2.3.4. $F_{*} \neq 0$ whenever $F \neq 0$; indeed the dimension of $F_{*}$ is at least as great as the dimension of $F$.

Proof. If $(G, E)$ is ergodic, then $N=[D]$ and so $[D] \cap F=0$. Then by proposition 1.1.6 orbits of $E$ contain at most one fixed point. Thus if $x \in R, K(x)$ contains precisely one fixed point. This gives 2 .

By proposition 2.1, $[D]=N$ is invariant under the action of $\boldsymbol{G}$ so that $R=N \oplus F$ is invariant under $\boldsymbol{G}$. Moreover if $\boldsymbol{g} x \in R$, then $K(\boldsymbol{g} x)$ contains a fixed point and since $K(x) \supset K(g x), K(x)$ contains the same fixed point. Thus $x \in R$.

Next we show that $R$ is closed. For $x \in R$, let $p(x)$ denote the unique fixed point in $K(x)$. Then $\|p(x)\| \leqq M\|x\|$ for all $x \in R$. Moreover, if $n \in N$ and $f \in F$, then $p(n+f)=f$ so that $\|f\|=\|p(n+f)\| \leqq$ $M\|n+f\|$.

Suppose then that $x_{n} \in R$ and that $x_{n} \rightarrow x_{0}$. Put $x_{n}=d_{n}+f_{n}$ where $d_{n} \in[D]=N$ and $f_{n} \in F$. Since $x_{n}$ is Cauchy and $\left\|f_{n}-f_{m}\right\| \leqq M \|\left(d_{n}-d_{m}\right)+$ $\left(f_{n}-f_{m}\right)\|=M\| x_{n}-x_{m} \|$, we conclude that $f_{n}$ is Cauchy. Thus $f_{n} \rightarrow f_{0} \in F$ and so $d_{n} \rightarrow x_{0}-f_{0} \in N$. Consequently $x_{0} \in N \oplus F=R$.

Let $x_{1}, x_{2} \in R$ and $x_{i}=n_{i}+f_{i}$ where $n_{i} \in N$ and $f_{i} \in F$. Then $p\left(x_{i}\right)=$ $f_{i}$ and $p\left(x_{1}+x_{2}\right)=p\left(\left(n_{1}+n_{2}\right)+\left(f_{1}+f_{2}\right)\right)=f_{1}+f_{2}$ since by Proposition $2.1 n_{1}+n_{2} \in N$. Thus $p$ is linear.

Finally in order to prove that $\operatorname{dim} F \leqq \operatorname{dim} F_{*}$, we may assume that $\operatorname{dim} F_{*}<\infty$. We then have $F_{*}=[D]^{\perp} \cong(E /[D])^{*}$ so that $\operatorname{dim} F_{*}=$ $\operatorname{dim}(E /[D])=\operatorname{codim}[D]$. But if $(G, E)$ is ergodic, $[D] \cap F=0$, and so in this case codim $[D] \geqq \operatorname{dim} F$.

This completes the proof.

Part 4 also follows from some results of Yood [15].

REMaRK. If $E$ is reflexive and both $(G, E)$ and $\left(G^{*}, E^{*}\right)$ are ergodic, (cf. Corollary 3.1.1) then applying Theorem 2.3.4 to both spaces, we conclude that $\operatorname{dim} F=\operatorname{dim} F_{*}$. In particular, this equality holds when $G$ is

${ }^{3}$ It is easy to see that $\boldsymbol{g} x \rightarrow x_{0}$ in the sense of Alaoglu-Birkhoff [1] if and only if $x \in R$ and $x_{0}=p(x)$. 
a group or an abelian semi-group on Hilbert space. When $G$ consists of contractions on Hilbert space, it follows for the same reason that $\operatorname{dim} F=\operatorname{dim} F_{*}$. But in this case one actually has more; namely $F-F_{*}$. For if $x \in F$, then $\|x\|^{2}=(g x, x)=\left(x, g^{*} x\right) \leqq\|x\|\left\|g^{*} x\right\| \leqq\|x\|^{2}$ and so $\|x\|^{2}=\left\|g^{*} x\right\|^{2}=\left(x, g^{*} x\right)$. Thus $\left\|g^{*} x-x\right\|^{2}=\left(g^{*} x-x, g^{*} x-x\right)=0$ so that $g^{*} x=x$ and $x \in F_{*}$.

3. Existence of fixed points. As the next proof will require us to conduct our arguments in the (non-normed) weak-*topology of $E^{*}$, we mention here some of the relevant background. The definitions and proofs of the results we use concerning topological vector spaces can be found in Bourbaki [3].

We define the weak-topology on a Banach space $E$ to be the least fine topology relative to which all the elements of the adjoint space $E^{*}$ are continuous. Thus the weak topology on the Banach space $E^{*}$ is defined using the elements of $E^{* *}$. By the weak-* topology on $E^{*}$ we mean the least fine topology relative to which the elements of $E$ induce continuous functionals. By definition then, when $E^{*}$ is endowed with the weak-* topology, any element of $E$ induces a continuous functional on it. One can show that every functional on $E^{*}$ which is continuous in the weak-* topology arises in this way from an element of $E$.

Although the norm topology is in general definitely richer in closed sets than the weak topology, Mazur's theorem asserts that a convex closed set is weakly closed. We will also make use of the fact that the unit ball of $E^{*}$ is compact in the weak-* topology. Finally, as the new topologies on $E$ and $E^{*}$ are locally convex (that is, every vector possesses a fundamental system of convex neighborhoods), the theorems of the Hahn-Banach type apply. These guarantee in particular the existence of continuous functionals strictly separating a given closed convex subset from a disjoint compact convex subset.

3.1 THEOREM. (a) If $(G, E)$ is ergodic, then any convex weak-* compact subset of $E^{*}$ which is invariant under the action of $G^{*}$, contains a fixed point. In particular, any orbit of $E^{*}$ which is compact in the weak-* topology contains a fixed point.

(b) If $\left(G^{*}, E^{*}\right)$ is ergodic, then any convex weakly-compact subset of $E$ which is invariant under the action of $G$, contains a fixed point. In particular, any orbit of $E$ which is compact in the weak topology contains a fixed point.

REMARK. Since by Mazur's theorem orbits are weakly closed, the two assertions of part (b) are actually equivalent. Orbits of $E^{*}$, on the other hand, need not be weak-* closed so that the first assertion 
of part (a) is really stronger than the second one.

Proof of the theorem. Part (a). Suppose that $W$ is a convex, weak-* compact subset of $E^{*}$ which is invariant under $G^{*}$ and yet which does not contain fixed points. Then $W \cap F_{*}=\phi$. As $F_{*}=D^{\perp}, F_{*}$ is weak-* closed. In the space $E^{*}$ endowed with the weak-* topology we may then apply one form of the Hahn-Banach Theorem to the disjoint closed convex set $F_{*}$ and the compact convex set $W$. This theorem asserts the existence of a weak-*-continuous functional on $E^{*}$ which strictly separates $W$ and $F_{*}$. As we have seen that all such functionals arise from elements in $E$, there is then an $x \neq 0$ in $E$ and a real number $\alpha$ with $\left(x, F_{*}\right)<\alpha<(x, W)$. Since $F_{*}$ is a subspace, this requires $\left(x, F_{*}\right)$ to be zero and so $x \in F_{*}^{\perp}=[D]$.

Thus $0<\alpha<(x, W)$ where $x \in[D]$. Choosing an arbitrary $\omega_{0} \in W$, we have $\boldsymbol{G}^{*} \omega_{0} \subset W$ so that $0<\alpha<\left(x, \boldsymbol{G}^{*} \omega_{0}\right)=\left(\boldsymbol{G} x, \omega_{0}\right)$ and hence $0<$ $\alpha \leqq\left(\overline{G x, \omega_{0}}\right)$. But as $\omega_{0}$ is continuous in the norm topology of $E$, $\left(K(x), \omega_{0}\right)=\left(\{\overline{\boldsymbol{G} x}\}, \omega_{0}\right) \subset\left(\overline{\boldsymbol{G} x, \omega_{0}}\right)$ and so $0<\alpha \leqq\left(K(x), \omega_{0}\right)$. In particular then, we conclude that $0 \notin K(x)$; that is, $x \notin N$. As $x$ was shown to be in $[D]$, this means that $(G, E)$ cannot be ergodic.

Part (b). Since the weak-* topology of $E^{* *}$ induces on $E$ (which is naturally imbedded in $E^{* *}$ ) a topology which coincides with the ordinary weak topology of $E$, a subset of $E$ which is weakly compact may be considered a subset of $E^{* *}$ which is compact in the weak-* topology on that space. An application of part (a) then gives part (b).

3.1.1 Corollary. If $E$ is reflexive, then $(G, E)$ is ergodic if and only if every orbit of $E^{*}$ contains at least one fixed point.

Proof. Orbits are bounded and (by Mazur's theorem) weakly closed so that in a reflexive space any orbit is weakly compact. Theorem 3.1 thus gives the forward implication. Example 2.2.4 gives the converse independently of reflexivity.

\subsection{REMARKS.}

3.2.1. If both $(G, E)$ and $\left(G^{*}, E^{*}\right)$ are ergodic, then any orbit in either $E$ or $E^{*}$ can contain at most one fixed point (Theorem 2.3.2). But then by Theorem 3.1, any weakly compact orbit of $E$ or any weak-* compact orbit of $E^{*}$ must contain precisely one fixed point. Since an abelian semi-group always acts ergodically, these results are valid in particular in the case where $G$ is abelian $\left(G^{*}\right.$ is also abelian). We shall see that the same is true when $G$ possesses a two-sided invariant mean (Remark 4.3.1.). 
3.2.2. By the corollary of the last theorem we see that when $E$ is reflexive every orbit contains precisely one fixed point if and only if

either (a) both $(G, E)$ and $\left(G^{*} E^{*}\right)$ are ergodic

or (b) every orbit of $E$ and of $E^{*}$ contains at least one fixed point.

In the case where $G$ is abelian, (a) is immediate. If $E$ is a Hilbert space and $G$ is either a semi-group of contractions or a (bounded) group, we have seen that (b) holds. In fact using the results of C. RyllNardzewski [14] mentioned in remark 1.5., (b) is valid on any reflexive Banach space so long as $G$ is a bounded group or if both $G$ and $G^{*}$ are each semi-groups of isometries. His proof presumably depends on delicate measure-theoretic machinery. It would be interesting to see if one could prove (a) directly in these cases.

4. Means and ergodicity. Let $E$ be the Banach space $B(\Omega)$ of all bounded continuous functions on the completely regular topological space $\Omega$ under sup norm ${ }^{4}$. An element $\xi \in E^{*}$ is called positive if $\xi(f) \geqq 0$ whenever $f \geqq 0$. In that case it is clear that $\|\xi\|=\xi(1)$. A positive functional $\lambda \in E^{*}$ is called a mean on $E$ if $\lambda(1)=1$. We then have $\|\lambda\|=1$ and moreover $|\lambda(f)| \leqq \lambda(|f|) \leqq\|f\|$ for any $f \in E$. Let $P$ be the set of means on $E$. Evidently $P$ is weak-* closed in $E^{*}$. As $P$ is convex and is contained in the (weak-* compact) unit ball of $E^{*}$, it follows that $P$ is weak-* compact.

An operator $T$ on $E$ is called an endomorphism (or a transitionoperator) if $T f \geqq 0$ whenever $f \geqq 0$ and also $T 1=1$. This is equivalent to requiring $T^{*} P \subset P$; i.e., that the set of means on $E$ is carried into itself by $T^{*}$. Finally as $\|f\| \leqq 1$ if and only if $-1 \leqq f \leqq 1$, we see that the norm of an endomorphism is 1 .

Suppose now that $G$ is a semi-group of endomorphisms on the Banach space $E$ as above. Since every element of $G$ has norm $1, G$ is bounded in the sense of our earlier discussion. A mean $\Lambda$ on $E$ is said to be invariant (under $G$ ) if $\Lambda(g f)=\Lambda(f)$ for each $g \in G, f \in E$. Thus an invariant mean on $E$ is simply an element of $P \cap F_{*}$. In general, of course, this set may be empty. However, as a consequence of Theorem 3.1, we have:

4.1 THEOREM. If $G$ is a semi-group of endomorphisms which acts ergodically on $E$, then $E$ possesses an invariant mean.

Proof. The set $P$ of means on $E$ is a convex weak-* compact subset of $E^{*}$. The fact that $G$ consists of endomorphisms means that $P$ is

${ }_{4}^{4}$ The development in this paragraph could be carried through taking for $E$ what Kakutani [11] has called an abstract $M$-space with unit, but his results show that the generality gained is only formal. 
carried into itself by every element of $G^{*}$. But then by Theorem 3.1 (a), $P$ contains a fixed point for $G$.

4.1.1. CoRollary. If $G$ is abelian, then E possesses an invariant mean (Kakutani [10] and Day [4]).

If the space $\Omega$ is a (topological) semi-group $G$, then there are several ways for $G$ to induce endomorphisms on the space $E=B(G)$. If $g \in G$ and $f \in B(G)$ let us define the operators $L_{\mathfrak{g}}$ and $R_{\mathfrak{g}}$ on $E$ by: $\left(L_{0} f\right)\left(g^{\prime}\right)=$ $f\left(g g^{\prime}\right)$ and $\left(R_{g} f\right)\left(g^{\prime}\right)=f\left(g^{\prime} g\right)$. We then have $L_{g_{1}} L_{g_{2}}=L_{g_{2} g_{1}}, R_{g_{1}} R_{g_{2}}=R_{g_{1} g_{2}}$ and $R_{g_{1}} L_{g_{2}}=L_{g_{2}} R_{g_{1}}$ so that $G_{L}=\left\{L_{g} ; g \in G\right\}$ and $G_{R}=\left\{R_{g} ; g \in G\right\}$ form two semi-groups of endomorphisms on $E$ which commute elementwise. Thus $G_{T}=\left\{L_{\theta_{1}} R_{g_{2}} ; g_{1}, g_{2} \in G\right\}$ also forms a semigroup of endomorphisms on $E$. Corresponding to these three semigroups we obtain the notion of left, right and two-sided invariant means on $E$ (or as we shall say, on $G$ ). By Corollary 4.1.1 any abelian semi-group possesses an invariant mean. The existence of Haar measure (cf. $\$ 4.4$ ) shows that any compact group possesses a (unique!) invariant mean.

Heretofore, in the discussion of bounded semi-groups of operators, the topology on the semi-group played no role. We might equally well have been dealing with an abstract semi-group $G$, together with a bounded representation of $G$ into the multiplicative semi-group of operators on $E$, where we call a representation $\pi$ of $G$ bounded when the image semi-group is bounded. If $G$ is a topological semi-group, we will say that the representation $\pi$ is weakly-continuous if $g \rightarrow(\pi(g) x, \xi)$ is a continuous function on $G$ for any $x \in E, \xi \in E^{*}$. For convenience we omit the letter $\pi$ and speak of the continuity of $(g x, \xi)$, the ergodicity of $(G, E)$, etc.

4.2 THeorem. Let $\pi$ be a bounded weakly-continuous representation of the topological semi-group $G$ on the Banach space $E$. Then if $G$ admits a right invariant mean, $(G, E)$ is ergodic.

Proof. For $x \in E, \xi \in E^{*}$, let $[x, \xi]$ denote the function in $B(G)$ whose value at $g$ is $(g x, \xi)$. If $\Lambda$ denotes a right invariant mean on $G$, then we may define a transformation $T: E \rightarrow E^{* *}$ by means of the equation $(T x, \xi)=\Lambda([x, \xi])$. Then

$$
\begin{aligned}
\|T x\| & =\sup _{|\xi| \mid 11}|(T x, \xi)|=\sup _{|| \xi|| \leqq 1}|\Lambda([x, \xi])| \leqq \sup _{|\xi \|| \leqq 1}\|[x, \xi]\| \\
& =\sup _{|| \xi|| \leqq 1} \sup _{g}|(g x, \xi)| \leqq M\|x\|
\end{aligned}
$$

Thus $T$ is continuous.

Observing that $R_{g}([x, \xi])=[g x, \xi]$ we have:

$$
(\operatorname{Tg} x, \xi)=\Lambda([g x, \xi])=\Lambda\left(R_{g}[x, \xi]\right)=\Lambda([x, \xi])=(T x, \xi)
$$


for any $\xi \in E^{*}$ so that $T g x=T x$ and consequntly $T$ vanishes on $D$. By the continuity of $T$ then, $T$ vanishes on $[D]$ and so a fortiori on $N$.

But now conversely if $T x=0$, we claim that $0 \in K(x)$ so that $x \in N$. For otherwise, we could find $\xi \in E^{*}$ and a real number $\alpha$ with $0<\alpha \leqq$ $(K(x), \xi)$. In particular $\alpha \leqq(g x, \xi)$ for all $g \in G$ and so $[x, \xi] \geqq \alpha$. But then $(T x, \xi)=\Lambda([x, \xi]) \geqq \alpha>0$, which contradicts the fact that $T x=0$. Thus $T x=0$ if and only if $x \in N$, so that $N$ is a linear subspace and $(G, E)$ is ergodic.

4.3.1. Let $G$ be a bounded semi-group of operators on $E$. If $G$ admits a right invariant mean when given either the discrete or the uniform operator topology then Theorem 4.2. applies so that $(G, E)$ is ergodic. If instead $G$ admits a left invariant mean in either of these topologies then $G^{*}$ admits a right invariant mean in the same topology so that $\left(G^{*}, E^{*}\right)$ is ergodic. Thus in this case by Theorem 3.1 any invariant compact convex set of $E$ contains a fixed point (cf. Day [6]). In particular if $G$ admits a two-sided invariant mean in either topology then any compact convex orbit of $E$ must contain precisely one fixed point.

4.3.2. Combining Theorem 4.1 and 4.2, we see that if $G$ is a (topological) semi-group of endomorphisms of the space $E=B(\Omega)$ (and $g \rightarrow(g x, \xi)$ is continuous) then whenever $G$ possesses a right invariant mean, $E$ also possesses a mean which is invariant under $G$.

\subsection{Application to Haar Measure on a Locally Compact Group.}

As an amusing application of the fact that abelian semi-groups admit invariant means, we give here a construction of Haar measure (or rather of a nontrivial invariant content ${ }^{5}$ ) on an arbitrary locally compact group.

Suppose then that $\mathscr{G}$ is a locally compact group and let $G$ denote the collection of neighbourhoods of the identity $e$ in $\mathscr{G}$. Then $G$ is an abelian semi-group under the operation of intersection! Let $\Lambda$ be an invariant mean on $G$. We wish to associate with each compact subset $K$ of the group $\mathscr{G}$ a bounded function $\widetilde{K}$ on $G$ in such a way that $\lambda: K \rightarrow \Lambda(\widetilde{K})$ will define a nontrivial invariant content. Let $K_{0}$ be a fixed compact neighborhood of $e$. Then if $S \subset \mathscr{G}$ and the interior of $S$ is nonvoid, define $(K: S)$ as the smallest integer $n$ such that $K$ can be covered by $n$ (left) translates of $S$. We now define the function $\widetilde{K}$ on $G$ by setting $\widetilde{K}(V)=(K: V) /\left(K_{0}: V\right)$ where $V \in G$. As $\widetilde{K}(V) \leqq\left(K: K_{0}\right)$, $\widetilde{K}$ is bounded and we may define $\lambda(K)=\Lambda(\widetilde{K})$. Observing that $(g K: V)=$ $(K: V)$ for $g \in \mathscr{G}$, we have $\widetilde{K}=(\widetilde{g K})$ and so $\lambda(g K)=\lambda(K)$. Also if $K$ has a nonvoid interior, then $K(V) \geqq 1 /\left(K_{0}: K\right)$ so that in this case

\footnotetext{
5 cf. Halmos-Measure Theory, Theorem B, p. 254.
} 
$\lambda(K) \geqq 1 /\left(K_{0}: K\right)>0$. It is clear that in general $\lambda\left(K_{1} \cup K_{2}\right) \leqq \lambda\left(K_{1}\right)+$ $\lambda\left(K_{2}\right)$. If, moreover, $K_{1}$ and $K_{2}$ are disjoint, $\left(K_{1} \cup K_{2}: V\right)=\left(K_{1}: V\right)+$ $\left(K_{2}: V\right)$ for all small enough $V \in G$. By virtue of the invariance of $\Lambda$, we then have $\lambda\left(K_{1} \cup K_{2}\right)=\lambda\left(K_{1}\right)+\lambda\left(K_{2}\right)$.

\section{BIBLIOGRAPHY}

1. L. Alaoglu and G. Birkhoff, General ergodic theorems, Ann. of Math., 41 (1940), 293-309.

2. G. Birkhoff, An ergodic theorem for general semi-groups, Proc. Nat. Acad. Sci. USA, 25 (1939), 625-627.

3. N. Bourbaki, Elements de Mathematique, Livre V. Espaces Vectorielles Topologiques, Paric (1953).

4. M.M. Day, Ergodic Theorems for Abelian semi-groups, Trans. Amer. Math Soc., 51 (1942), 399-412.

5. - Reflexive Banach spaces not isomorphic to uniformly convex spaces, Bull. Amer. Math. Soc., 47 (1941), 313-317

6. - - Fixed-point theorems for compact convex sets, Ill. J. Math., 5 (1961), 585-590.

7. J. Dixmier, Les Moyennes invariantes dans les semi-groupes et leurs applications, Acta Scientif. Math., 12 (1950).

8. W.F. Eberlein, Abstract ergodic theorems and wealkly almost periodic functions, Trans. Amer. Math. Soc., 67 (1949), 217-240.

9. K. Jacobs, Ein Ergodensatz fur beschrankte Gruppen im Hilbertschen Raum, Math. Ann., 128 (1954), 340-349.

10. S. Kakutani, Two fixed point theorems concerning bicompact convex sets. Proc. Imp. Acad. Tokyo, 14 (1938), 242-245.

11. - Concrete representation of abstract $M$-spaces, Ann. Math., 42 (1941), 994-1024.

12. K. deLeeuw and I. Glicksberg, Applications of almost periodic compactications, Acta Mathematica 105 (1961), 63-97.

13. J.E.L. Peck, An ergodic theorem for a non-commutative semi-group of linear operators, Proc. Amer. Math. Soc., 2 (1951), 414-421.

14. C. Ryll-Nardzewski, Generalized random ergodic theorems and weakly almost periodic functions (to appear in Studia Math. and elsewhere).

15. B. Yood, On fixed points for semi-groups of linear operators, Proc. Amer. Math. Soc., 2 (1951), 225-233. 



\section{PACIFIC JOURNAL OF MATHEMATICS}

\section{EDITORS}

\author{
Ralph S. Phillips \\ Stanford University \\ Stanford, California \\ M. G. Arsove \\ University of Washington \\ Seattle 5 , Washington
}

\author{
A. L. Whiteman \\ University of Southern California \\ Los Angeles 7, California \\ Lowell J. Paige \\ University of California \\ Los Angeles 24, California
}
E. F. BECKENBACH
D. DERRY
ASSOCIATE EDITORS
T. M. CHERRY
M. OHTSUKA
H. L. ROYDEN
E. G. STRAUS
E. SPANIER
F. WOLF

\section{SUPPORTING INSTITUTIONS}

\author{
UNIVERSITY OF BRITISH COLUMBIA \\ CALIFORNIA INSTITUTE OF TECHNOLOGY \\ UNIVERSITY OF CALIFORNIA \\ MONTANA STATE UNIVERSITY \\ UNIVERSITY OF NEVADA \\ NEW MEXICO STATE UNIVERSITY \\ OREGON STATE UNIVERSITY \\ UNIVERSITY OF OREGON \\ OSAKA UNIVERSITY \\ UNIVERSITY OF SOUTHERN CALIFORNIA
}

\author{
STANFORD UNIVERSITY \\ UNIVERSITY OF TOKYO \\ UNIVERSITY OF UTAH \\ WASHINGTON STATE UNIVERSITY \\ UNIVERSITY OF WASHINGTON \\ AMERICAN MATHEMATICAL SOCIETY \\ CALIFORNIA RESEARCH CORPORATION \\ SPACE TECHNOLOGY LABORATORIES \\ NAVAL ORDNANCE TEST STATION
}

Mathematical papers intended for publication in the Pacific Journal of Mathematıcs should be typewritten (double spaced), and the author should keep a complete copy. Manuscripts may be sent to any one of the four editors. All other communications to the editors should be addressed to the managing editor, L. J. Paige at the University of California, Los Angeles 24, California.

50 reprints per author of each article are furnished free of charge; additional copies may be obtained at cost in multiples of 50 .

The Pacific Journal of Mathematics is published quarterly, in March, June, September, and December. Effective with Volume 13 the price per volume (4 numbers) is $\$ 18.00$; single issues, $\$ 5.00$. Special price for current issues to individual faculty members of supporting institutions and to individual members of the American Mathematical Society: $\$ 8.00$ per volume; single issues $\$ 2.50$. Back numbers are available.

Subscriptions, orders for back numbers, and changes of address should be sent to Pacific Journal of Mathematics, 103 Highland Boulevard, Berkeley 8, California.

Printed at Kokusai Bunken Insatsusha (International Academic Printing Co., Ltd.), No. 6, 2-chome, Fujimi-cho, Chiyoda-ku, Tokyo, Japan.

PUBLISHED BY PACIFIC JOURNAL OF MATHEMATICS, A NON-PROFIT CORPORATION

The Supporting Institutions listed above contribute to the cost of publication of this Journal, but they are not owners or publishers and have no responsibility for its content or policies. 


\section{Pacific Journal of Mathematics}

\section{Vol. 12 , No. 4 \\ April, 1962}

Tsuyoshi Andô, On fundamental properties of a Banach space with a cone ..... . 1163

Sterling K. Berberian, A note on hyponormal operators ................ 1171

Errett Albert Bishop, Analytic functions with values in a Frechet space . . . . . . . 1177

(Sherman) Elwood Bohn, Equicontinuity of solutions of a quasi-linear equation ............................................ 1193

Andrew Michael Bruckner and E. Ostrow, Some function classes related to the class of convex functions . . . . . . . . . . . . . . . . . . . . . . . . 1203

J. H. Curtiss, Limits and bounds for divided differences on a Jordan curve in the complex domain . . ................................. 1217

P. H. Doyle, III and John Gilbert Hocking, Dimensional invertibility . . . . . . . . 1235

David G. Feingold and Richard Steven Varga, Block diagonally dominant matrices and generalizations of the Gerschgorin circle theorem ................. 1241

Leonard Dubois Fountain and Lloyd Kenneth Jackson, A generalized solution of the boundary value problem for $y^{\prime \prime}=f\left(x, y, y^{\prime}\right) \ldots \ldots \ldots \ldots \ldots \ldots \ldots \ldots \ldots$

Robert William Gilmer, Jr., Rings in which semi-primary ideals are primary. . . . . 1273

Ruth Goodman, K-polar polynomials .......................... 1277

Israel Halperin and Maria Wonenburger, On the additivity of lattice completeness ........................................... 1289

Robert Winship Heath, Arc-wise connectedness in semi-metric spaces . . . . . . . . 1301

Isidore Heller and Alan Jerome Hoffman, On unimodular matrices . . . . . . . . . . . 1321

Robert G. Heyneman, Duality in general ergodic theory . . . . . . . . . . . . . . . 1329

Charles Ray Hobby, Abelian subgroups of p-groups . . . . . . . . . . . . . . . 1343

Kenneth Myron Hoffman and Hugo Rossi, The minimum boundary for an analytic

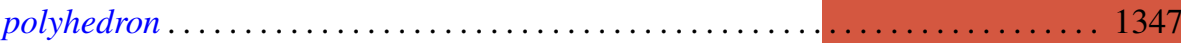

Adam Koranyi, The Bergman kernel function for tubes over convex cones ........ 1355

Pesi Rustom Masani and Jack Max Robertson, The time-domain analysis of a continuous parameter weakly stationary stochastic proces.

William Schumacher Massey, Non-existence of almost-complex structures on

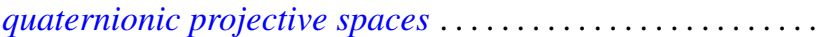

Deane Montgomery and Chung-Tao Yang, A theorem on the action of $\mathrm{SO}(3) \ldots . .1385$

Ronald John Nunke, A note on Abelian group extensions . . . . . . . . . . . . . 1401

Carl Mark Pearcy, A complete set of unitary invariants for operators generating finite $W^{*}$-algebras of type $I$

Edward C. Posner, Integral closure of rings of solutions of linear differential equations.

Duane Sather, Asymptotics. III. Stationary phase for two parameters with an application to Bessel functions.

J. Śladkowska, Bounds of analytic functions of two complex variables in domains

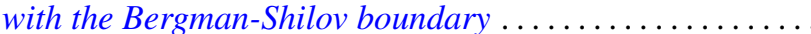

Joseph Gail Stampfli, Hyponormal operators .

George Gustave Weill, Some extremal properties of linear combinations of kernels

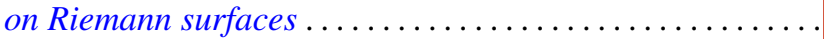

